\title{
FULL SCALE MONITORING OF THE TWIN CHIMNEYS OF THE ROVINARI POWER PLANT
}

\author{
I. Bayati, M. Belloli ${ }^{\text {a }}$, L. Rosa, A. Zasso \\ Politecnico di Milano, Department of Mechanical Engineering, via G. La Masa 1, 20156 Milan, Italy
}

\begin{abstract}
The presented paper deals with the structural identification and monitoring of two twin chimneys in very close arrangement. Due to twin arrangement, important interference effects are expected to modify the chimney response to wind action, causing vortex shedding and state-dependent excitation associated to the oscillatory motion of the leeward chimney, in and out of the windward chimney wake. The complexity of the physics of this problem is increased by the dependency of the aerodynamics of circular cylinders on Reynolds number; however, there is a weakness of literature about cylinders behaviour at critical and super-critical range of Reynolds number, due to experimental limitations. Also the International Committee on Industrial Chimneys (CICIND) does not provide, at present, any specific technical guideline about twin chimneys whose interaxis distance is less or equal two times the diameter, as in this case. For this reason a Tuned Mass Damper (TMD) has been installed in order to increase the damping of the chimney, as merely suggested. This work aims at assessing the effectiveness of the installed TMD and characterizing the tower dynamic behaviour itself due to the wind excitation, as well as providing full scale measurements for twin cylinders configuration at high Reynolds numbers.
\end{abstract}

\section{Introduction}

This work deals with the structural identification and monitoring of one of two chimneys of the Rovinari Power Plant, in Romania, that have been erected as part of coal-fired boiler units retrofitting. The new chimneys are erected in twin arrangement, so each pair of chimneys represent a "twin cylinder" configuration, from an aerodynamic point of view, since the axle spacing between the chimneys is smaller than three times their diameter, Figure 1.

Important interference effects are expected to modify the chimney response to wind action. The main effects are an increase of the vortex shedding excitation and a relevant and usually dangerous state-dependent excitation associated to the oscillatory motion of the leeward chimney in and out of the windward chimney wake, [1]. The great difference with the "no interference" counterpart, is that the dynamic excitation of the leeward structure is given by a drag and lift force depending on the position (aerodynamic stiffness term) and on the velocity (aerodynamic damping term) of the leeward chimney moving within the wake of the windward chimney, acting as a state-dependent excitation. It is well known that the warning on state-dependent forcing comes from the potentially dangerous dynamic instability phenomena associated to which could be added the usual dynamic excitation due to vortex shedding, although the associated phenomenology is more complex because of the wake interaction of the two chimneys [2-3]. For this reason, when it comes to consider the wind excitation on these structures, wake effects must be taken into account, as they determine the coupled behavior of the twin structures. Nevertheless, the complexity of the physics of this problem is augmented by the well-known dependency of the aerodynamics of circular cylinders on Reynolds number: because of the practical limitations which come into place in carrying out experimental measurements in critical and super-critical range of Reynolds number, only for sub-critical range a large amount of data is available in regards to the aerodynamics of twin cylinders [4-5-6-7-8].

The parameter strictly linked to the aerodynamic response of a structure is the Scruton Number, defined as:

$$
S c=\frac{4 \pi m_{e} \xi}{\rho D^{2}}
$$

where $m_{e}$ is the mass per unit of length, $\xi$ is the nondimensional damping and $D$ a characteristic dimension of the structure, $\rho$ is the density of the air. The risk of violent vortex induced vibrations is linked to the Scruton Number. Experience shows that there is no risk of lock-in if $S c>20$. On the other hand if $S c<10$, the risk of lock-in is pronounced, [9].

At present, the International Committee on Industrial Chimneys (CICIND) does not provide any specific recommendations on twin chimneys whose interaxis distance is less or equal two times the diameter, but a very clear warning is given concerning this type of configuration, recommending addition of structural

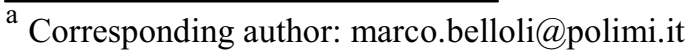


damping to increase the chimney's Scruton Number to more than 25, [10].

Being the Scruton Number the dominant parameter for the aerodynamic structure interaction, a Tuned Mass Damper (TMD) was adopted for increasing the chimney Scruton Number, with respect to the free standing configuration. Not many examples of "twin chimneys" can be found in literature, nevertheless a quite similar study confirmed the mitigation of the wind-induced vibrations reached by installing a TMD device similar to those installed at Rovinari Power Station, [11]. Figure 2 shows the TMD after its installation one chimney.

This work aims to estimate the dynamic characteristic of the chimney, verifying the differences between the dynamics of design and the built structure. Furthermore the dynamic characteristic of the installed TMD were identified. In order to characterize the chimney's dynamics both as a free standing system with and without the assistance of the TMD, in terms of damping ratio, some tests were performed in two configurations: "TMD Blocked" (i.e. preventing the relative motion ChimneyTMD through high stiffness constraints) and "TMD inservice" (i.e. Chimney-TMD relative motion allowed, with the TMD device acting as a pendulum with the related design properties).

\section{Structural characteristics}

Table 1 shows the chimney global geometry and its natural frequencies. The theoretical values were calculated using a FE model, whereras the experimental frequencies were measured on-site before the installation of the TMD. The FE model was developed by CRI International while these measurements were carried out by the Universitatea Tehnica de Constructii Bucuresti which was also in charge of giving the specification of the TMD, shown in Table 2.

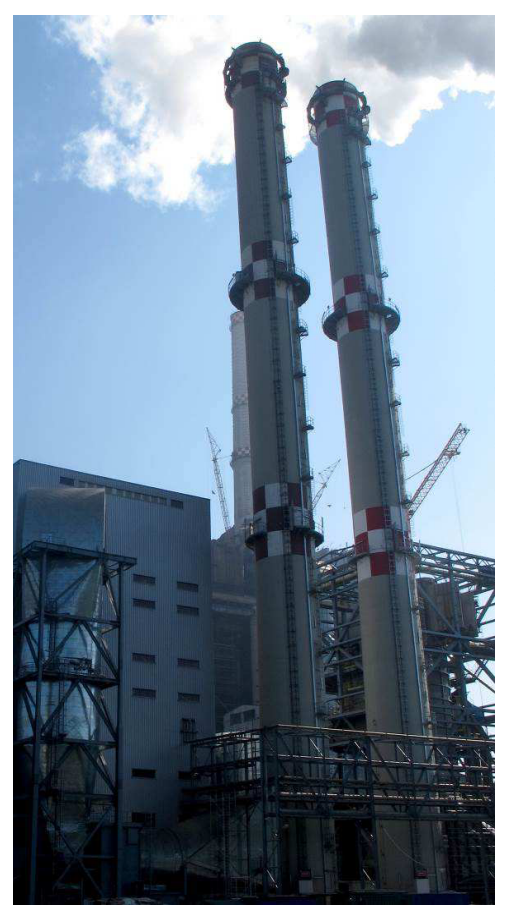

Figure 1. The new twin chinmeys at Rovinari Power Plant.

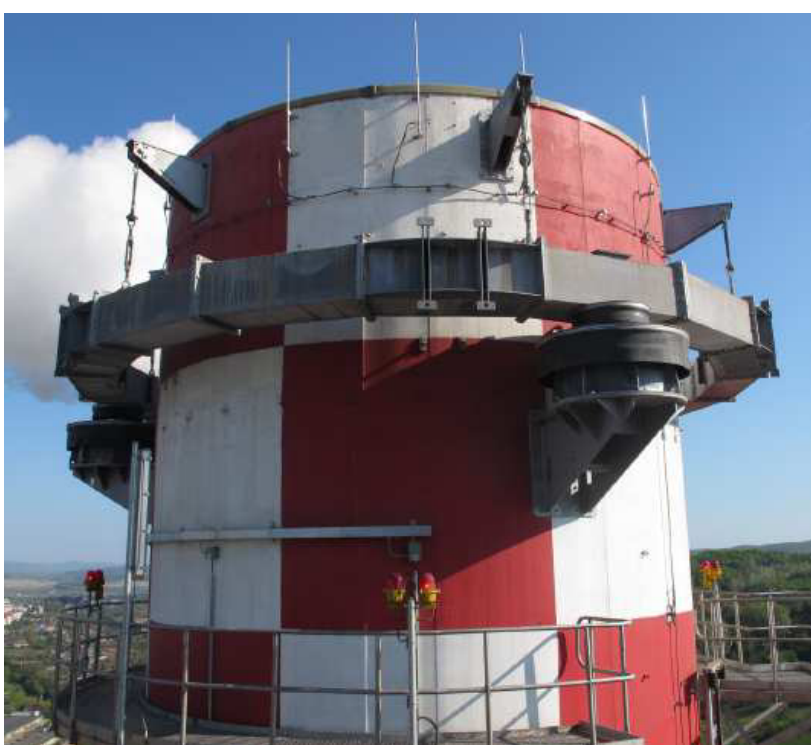

Figure 2. The tuned mass damper after its installation.

Table 1. Chimney global geometry and dynamic characteristic.

\begin{tabular}{|c|c|}
\hline Chimney height & $H=120 \mathrm{~m}$ \\
\hline Chimney total mass & $M=1.793 \mathrm{e} 6 \mathrm{~kg}$ \\
\hline Base/Top outer diameter & $\begin{array}{c}8.25 \mathrm{~m} / 7.42 \mathrm{~m} \text { (from } \\
+40 \mathrm{~m} \text { unchanged) }\end{array}$ \\
\hline $\begin{array}{c}\text { First vibration mode: } \\
\text { FEmodel / Experimental }\end{array}$ & $0.40 \mathrm{~Hz} / 0.41 \mathrm{~Hz}$ \\
\hline $\begin{array}{c}\text { Second vibration mode: } \\
\text { FEmodel / Experimental }\end{array}$ & $2.23 \mathrm{~Hz} / 2.01 \mathrm{~Hz}$ \\
\hline
\end{tabular}

Table 2. Specification of the TMD system.

\begin{tabular}{|c|c|}
\hline Effective TMD mass & $M_{t m d}=28.2 \mathrm{e} 3 \mathrm{~kg}$ \\
\hline Tuning frequency range & $f=0.38-0.44 \mathrm{~Hz}$ \\
\hline Internal damping ratio & $\xi_{t m d}=8-10 \%$ \\
\hline Operation Amplitude & $80 \mathrm{~mm}$ \\
\hline
\end{tabular}

\section{Experimental set-up}

The monitoring system was able to measure the wind excitation, through an anemometer, and the dynamic response of the chimney to the environmental forcing. Due to the symmetry of the structures, only one chimney was instrumented. The dynamic behaviour of the chimney was monitored using four ICP accelerometers with a sensitivity of $1000 \mathrm{mV} / \mathrm{g}$ in a frequency range from $0.025 \mathrm{~Hz}$ to $800 \mathrm{~Hz}( \pm 5 \%)$ and a measurement range $2.5 \mathrm{~g}$ pk. Two sensors (Accl and Acc4) were installed on the top of the structure and disposed in two orthogonal directions in order to measure the horizontal displacements in the plan defined by the two accelerometers. Other two accelerometers (Acc2 and Acc3) were installed on the TMD, at the corresponding position of the accelerometers on the chimney, in order to measure the TMD oscillations. The experimental set-up is shown in Figure 3. 


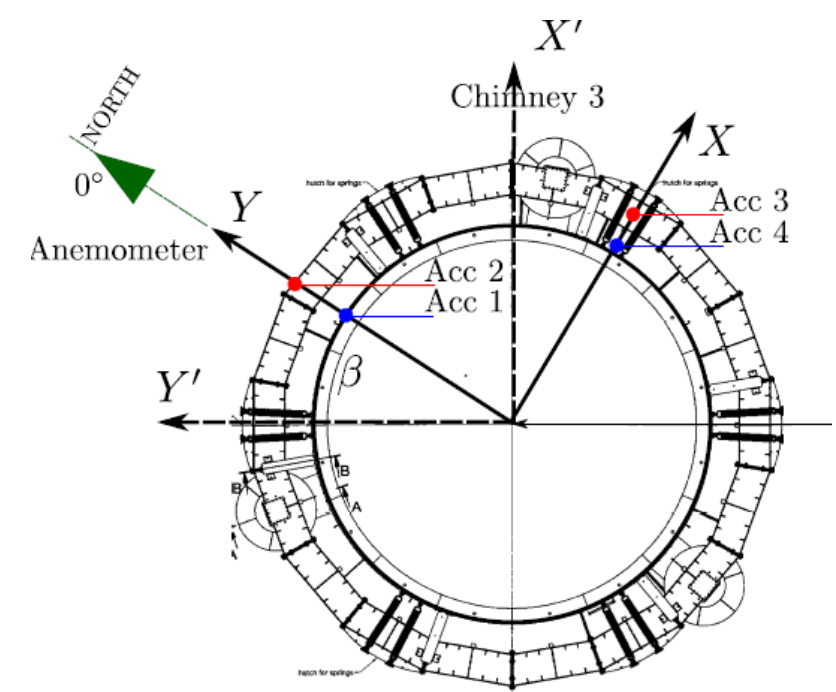

Figure 3. Experimental set-up on chimney 3.

\section{Experimental tests}

\subsection{Dynamic characterization of the TMD}

The first step was the definition of the dynamic characteristics of the TMD as well as the structural damping related to the coupled system Chimney-TMD. In order to gather this information, the test procedure consisted in manually provide an imposed displacement to the TMD itself and then allow it to vibrate free while acquiring the signals. A special attention was put to perform these decay tests at very low wind speed in order not to gather misleading measurements.

Figure 4 shows, as an example, a decay after having imposed to the TMD an initial displacement in ydirection $(A c c 2)$. The time history is quoted in $\left(\mathrm{m} / \mathrm{s}^{2}\right)$ and it was filtered using a $2 \mathrm{~Hz}$ low-pass filter. The decay tests were used to compute the natural frequency $f_{\text {tmd }}$ by the Fourier Transform and the non-dimensional damping of the coupled system Chimney-TMD $\xi_{\text {tmd }}$ using the Hilbert method. Table 3 reports the output of this analysis: as clearly visible the dynamic behaviour of the TMD is uniform in the plane.

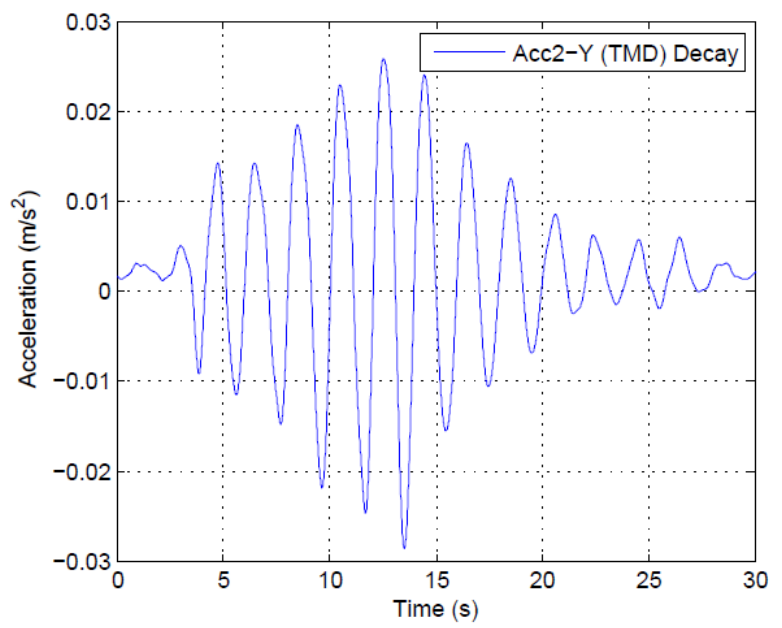

Figure 4. Decay TMD accelerometer Acc2-Y.
Table 3. TMD experimental characteristics.

\begin{tabular}{|c|c|c|}
\hline Acc2-y & $f_{\text {tmd }}=0.507 \mathrm{~Hz}$ & $\xi_{\text {tmd }} \sim 6 \%$ \\
\hline Acc3-x & $f_{\text {tmd }}=0.510 \mathrm{~Hz}$ & $\xi_{\text {tmd }} \sim 6 \%$ \\
\hline
\end{tabular}

\subsection{Dynamic characterization of the chimney with TMD blocked: natural frequencies}

In order to characterize the chimney as a free standing system with no effect due to the TMD operating some tests were carried out blocking the TMD (i.e. preventing the relative motion between the chimney and the TMD) by means of high stiffness constraints. In the following, these tests are referred to as "TMD blocked", whereas the design configuration allowing relative motion between the chimney and the TMD, consistently with its pendulum properties, are referred to as "TMD inservice".

The measurements during the most windy day in the experimental campaign were considered in the analysis in order to have an acceptable excitation of the structure. During these measurements the instrumented chimney was always in windward position.

Figure 5 shows the FFT estimated considering the total length of the measurements, equal to $10800 \mathrm{~s}$, divided and averaged in order to improve the signal to noise ratio, resulting in a frequency resolution of $\Delta \mathrm{f}=0.0014 \mathrm{~Hz}$. The analysis is focused on the first natural frequency of the chimney, being the mode mainly protected by the TMD and the mostly affecting the dynamic of the structure. Both the FFT of the Acc1-Y acceleration component, Figure 5(a) and the FFT of the Acc $4-X$ acceleration component, Figure 5(b), show two very close peaks, with $f=0.382 \mathrm{~Hz}$ and $f=0.403 \mathrm{~Hz}$.

Two closely located dominant frequencies indicates that the structure of the chimney is inevitably imperfectly axisymmetric. This is probably caused by an imperfect geometry, heterogeneous material properties, plants installed (i.e. the lift or the TMD itself) or heterogeneous foundations.

Previous research on similar structure showed that the bisymmetry of a structure should be taken into account for a reliable evaluation of its structural damping, [14]. Subsequently the direction of evaluation of the vibration of the chimney has a direct influence on the evaluation of the structural damping and to its dynamics. In particular the most reliable value of the structural damping can be obtained considering the vibrations along the symmetry axes of the chimney. For this reason the coordinate system $\mathrm{X}-\mathrm{Y}$, associated to the direction of the accelerometers Acc1, and Acc4, Figure 3, was rotated in order to analysed the vibrations along others directions. The accelerations components along the new directions $\mathrm{X}^{\prime}-\mathrm{Y}^{\prime}$ were subsequently calculated, based on the following transformation of coordinates:

$$
\begin{aligned}
& A c c 1-Y^{\prime}=A c c 1 \cos \beta-A c c \sin \beta \\
& A c c 4-X^{\prime}=A c c 1 \sin \beta+A c c \cos \beta
\end{aligned}
$$



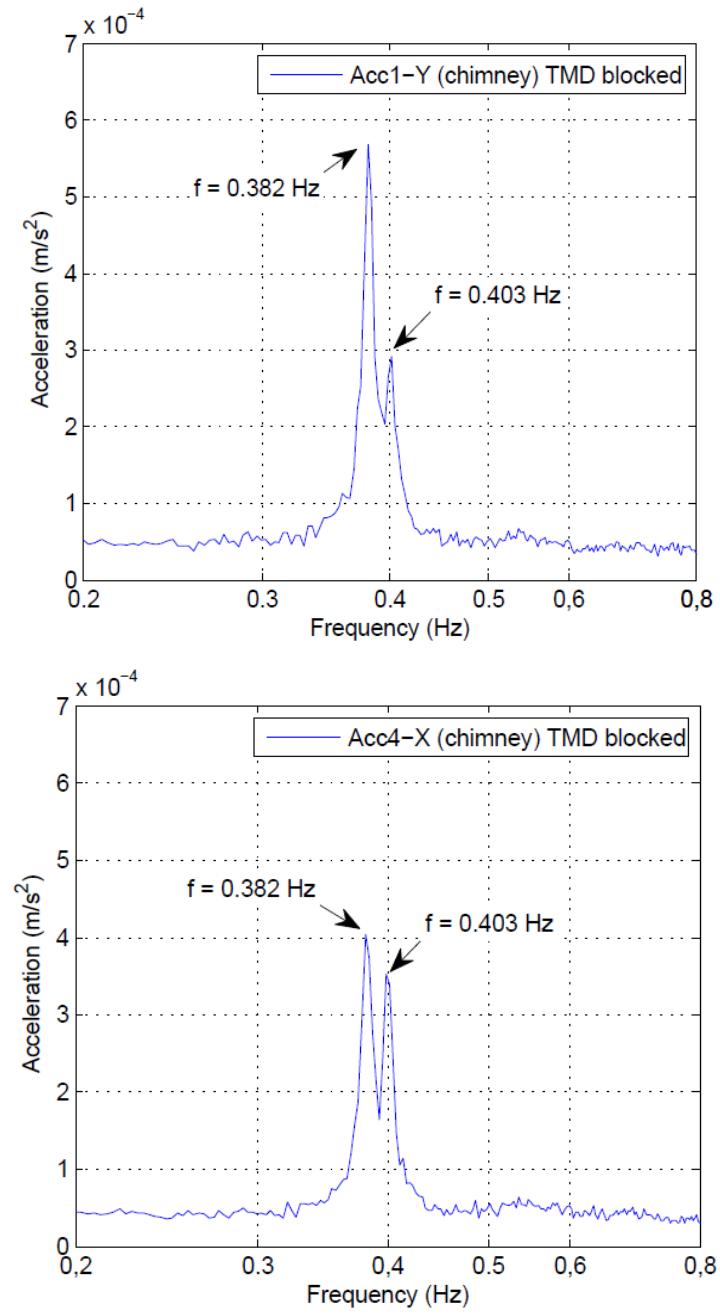

(a)

Figure 5. FFT of accelerometers on the chimney in TMD blocked configuration. Reference system $\mathrm{X}-\mathrm{Y}$.

being $\beta$ the rotation angle of the new coordinate system $\mathrm{X}^{\prime}-\mathrm{Y}^{\prime}$ related to the original coordinate system $\mathrm{X}-\mathrm{Y}$, see Figure 3.

The measurements were subsequently analyzed for different rotation of the reference system $\mathrm{X}^{\prime}-\mathrm{Y}^{\prime}$, considering a rotation angle $0^{\circ}<\beta<90^{\circ}$.

Figure 6 shows the FFT of the accelerometers obtained considering an angle of rotation $\beta=38^{\circ}$ : as one can see each direction shows one dominant frequency, $f_{X^{\prime}}=0.403 \mathrm{~Hz}$ and $f_{Y^{\prime}}=0.382 \mathrm{~Hz}$ respectively, confirming that the chimney has a bisymmetric structure and that the directions marked by the axes $X^{\prime}-Y^{\prime}$ and $\beta=38^{\circ}$ can be considered as symmetry axes of the chimney.

\subsection{Dynamic characterization of the chimney with TMD blocked: structural damping}

The wind force acting on a structure provides a great amount of energy and allows to properly excite the chimney. The wind force is unknown, but this kind of excitation can be considered very similar to a random process in the frequency range investigated. The identification of the non-dimensional damping can therefore be performed using an output-only approach: in

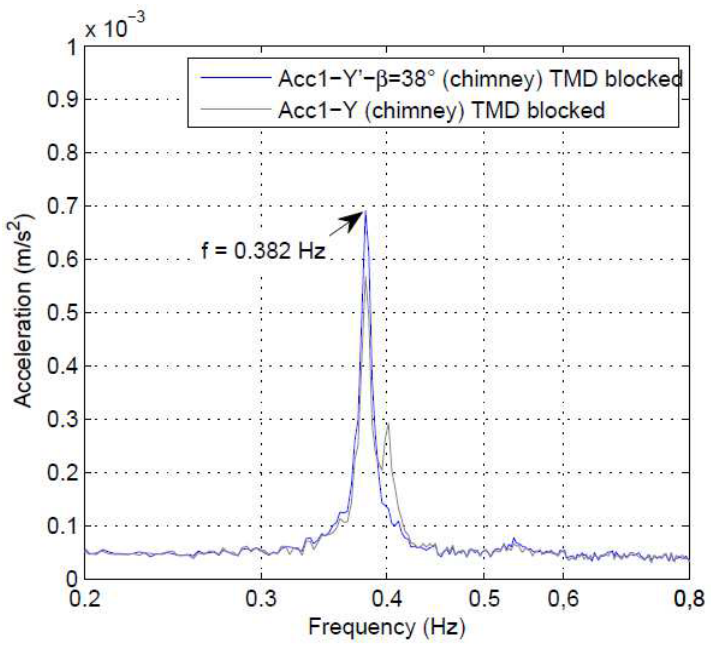

(a)

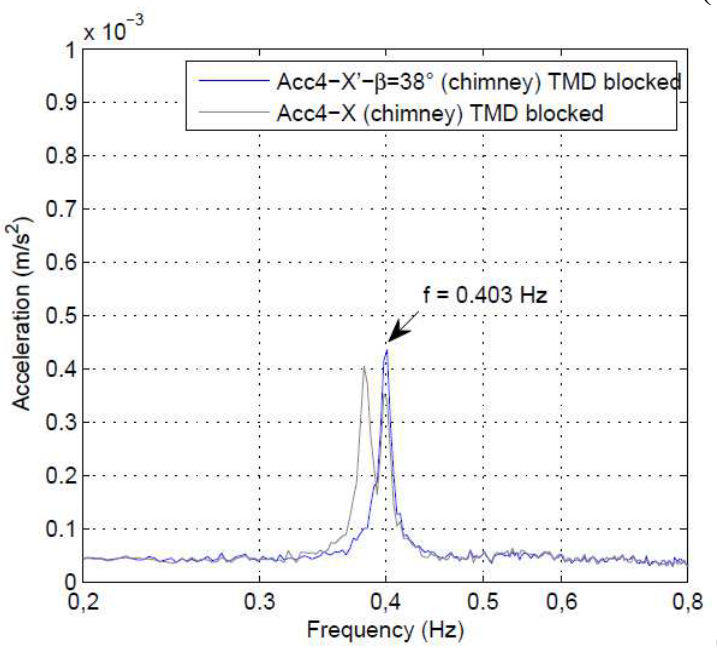

Figure 6. FFT of accelerometers on the chimney in TMD blocked configuration. Reference system $X^{\prime}-Y^{\prime}, \beta=38^{\circ}$. (a) FFT of Acc1-Y'. (b) FFT of Acc4-X'.

the present study the random decrement technique (RDT) was adopted along with the Hilbert method, [15]. A fourth-order Butterworth pass-band digital filter with band-pass $0.2 \mathrm{~Hz}<f_{\text {band }}<0.5 \mathrm{~Hz}$ was used. The trigger condition for the RDT was set equal to $x_{0}=0.5 \sigma$ being $\sigma$ the standard deviation of the filtered acceleration time history, resulting in more than 2500 time segments taken into account in the averaging process of the $\mathrm{RD}$ technique.

Figure 8 shows the RD signatures of accelerometers on the chimney in the reference system $\mathrm{X}-\mathrm{Y}, \beta=0^{\circ}$. As one can see the RD signatures appear incorrect with considerable beating phenomenon. These elaborations, associated to spectra with the two very close peaks in frequencies (Figure 5) cannot be used to estimate correctly the structural damping.

The RDT was then applied on the filtered accelerations of the chimney along the symmetry axes $X^{\prime}$ $-\mathrm{Y}^{\prime}, \beta=38^{\circ}$.

Figure 8 shows the RD signatures of accelerometers on the chimney in this reference system. As one can see the figures do not show beating phenomenon which could appear if the principal directions are not considered and allow to correctly estimate the structural damping using the Hilbert Method. 


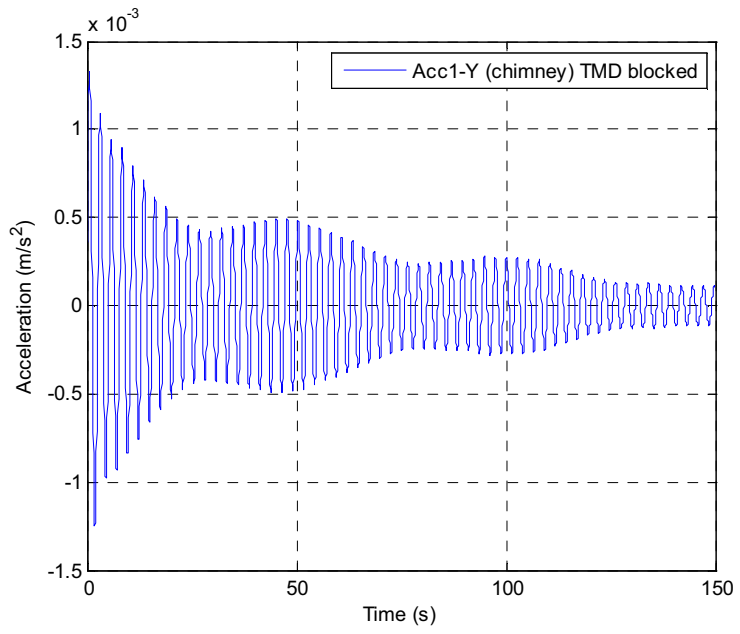

(a)

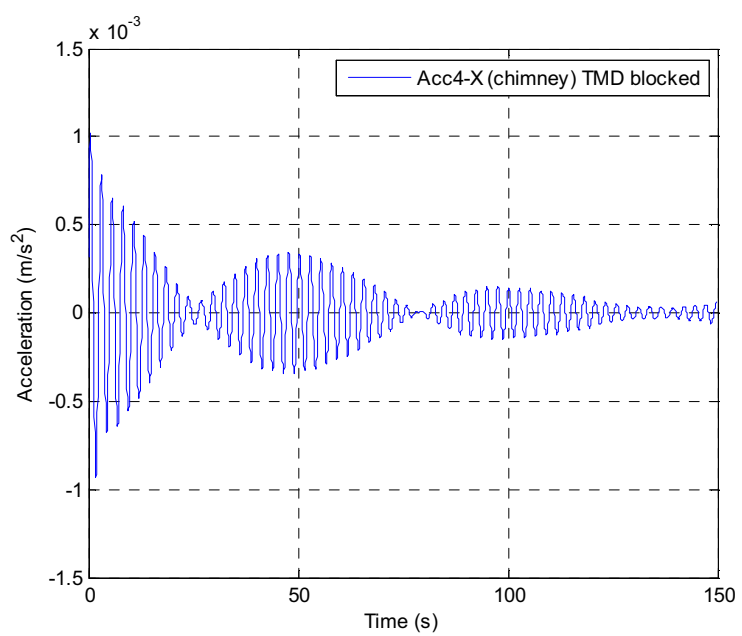

(b)

Figure 7. RD signatures of accelerometers on the chimney in TMD blocked configuration. Reference system $X-Y, \beta=0^{\circ}$.

(a) Signature of Acc1-Y. (b) Signature of Acc4-X.

The values of the damping ratios estimated along the two principal axes of the chimney are shown in the figures, corresponding to $\xi_{Y^{\prime}}=0.60 \%$ and $\xi_{X^{\prime}}=0.72 \%$, respectively associated to the frequency $f_{Y^{\prime}}=0.382 \mathrm{~Hz}$ and $f_{X^{\prime}}=0.403 \mathrm{~Hz}$.

It could be also noted that the higher natural frequencies before the installation of the TMD shown in Table 2 are due to lower mass at the top of the chimney.

\subsection{Dynamic characterization of the chimney with TMD in-service: natural frequencies and structural damping}

The dynamic characterization of the chimney with the TMD in-service took place on several days in order to measure data with different wind direction and to collect possible interference effects between the twin chimneys. The analysis of the data was done using the RD technique with the same parameters described in the previous paragraph. The analysis were always carried out concatenating several time histories in order to have an important excitation of the structure.

As an example of the results, Figure 9 shows the results of the analysis with wind generally coming from East, leading the chimney to be leeward but never in the wake of to the twin counterpart.

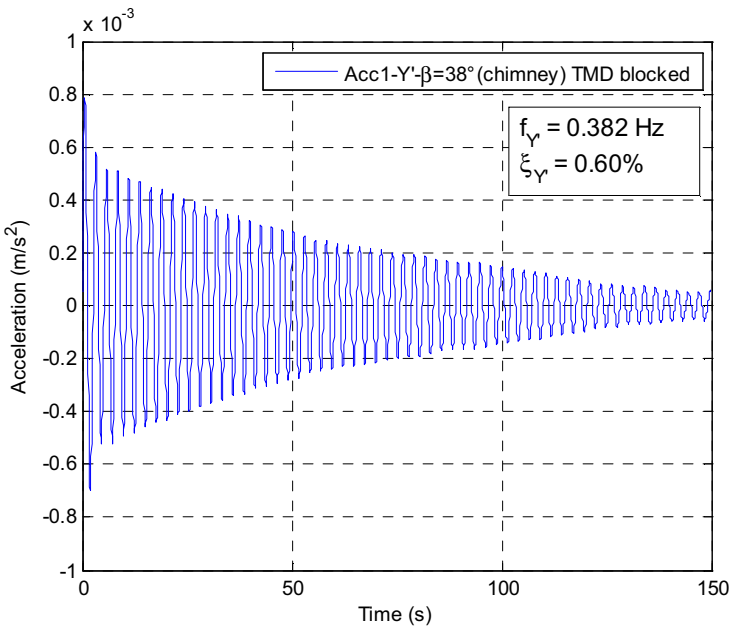

(a)

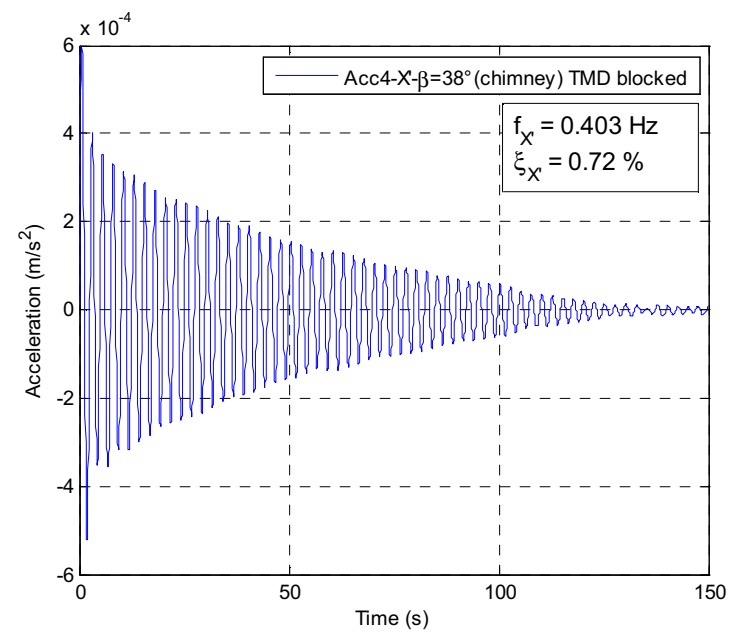

Figure 8. RD signatures of accelerometers on the chimney in TMD blocked configuration. Reference system $X^{\prime}-Y^{\prime}, \beta=38^{\circ}$. (a) Signature of Acc1-Y'. (b) Signature of Acc4-X'.

The figure shows the wind conditions during the tests, in terms of thirty minutes interval mean wind speed and mean direction; the FFT of the accelerometers on the chimney along the principal axes of the chimney $\mathrm{X}^{\prime}-\mathrm{Y}^{\prime}$ $\beta=38^{\circ}$, with frequency resolution at least equal to $\triangle \mathrm{f}=0.0014 \mathrm{~Hz}$; the $\mathrm{RD}$ signatures of the same accelerometers.

The values of the damping ratios estimated along the two principal axes of the chimney, correspond to $\xi_{Y^{\prime}}=$ $1.60 \%$ and $\xi_{X^{\prime}}=2.1 \%$, respectively associated to the frequency $f_{Y^{\prime}}=0.366 \mathrm{~Hz}$ and $f_{X^{\prime}}=0.380 \mathrm{~Hz}$.

Considering the spectra of the acceleration signals shown in Figure 6, one can see the slight shift in frequencies compared to the case of TMD in-service, see Figure 9(b). In particular the frequencies decrease from the values $f_{Y}=0.382 \mathrm{~Hz}$ and $f_{X^{\prime}}=0.403 \mathrm{~Hz}$ in "TMD blocked" configuration to $f_{Y^{\prime}}=0.367 \mathrm{~Hz}$ and $f_{X^{\prime}}=0.377 \mathrm{~Hz}$ whenever the TMD is free to vibrate (In-service). This shift in frequency between the "TMD Blocked" (higher frequency peak) and "TMD In-service"(lower frequency peak) configurations across the first natural frequency of the structure is typical of a two-degrees of freedom equivalent for a TMD-Structure coupled system of the mode considered. These observations are consistent with effectiveness of the adoption of a TMD solution for softening the vibrations of the chimney. 

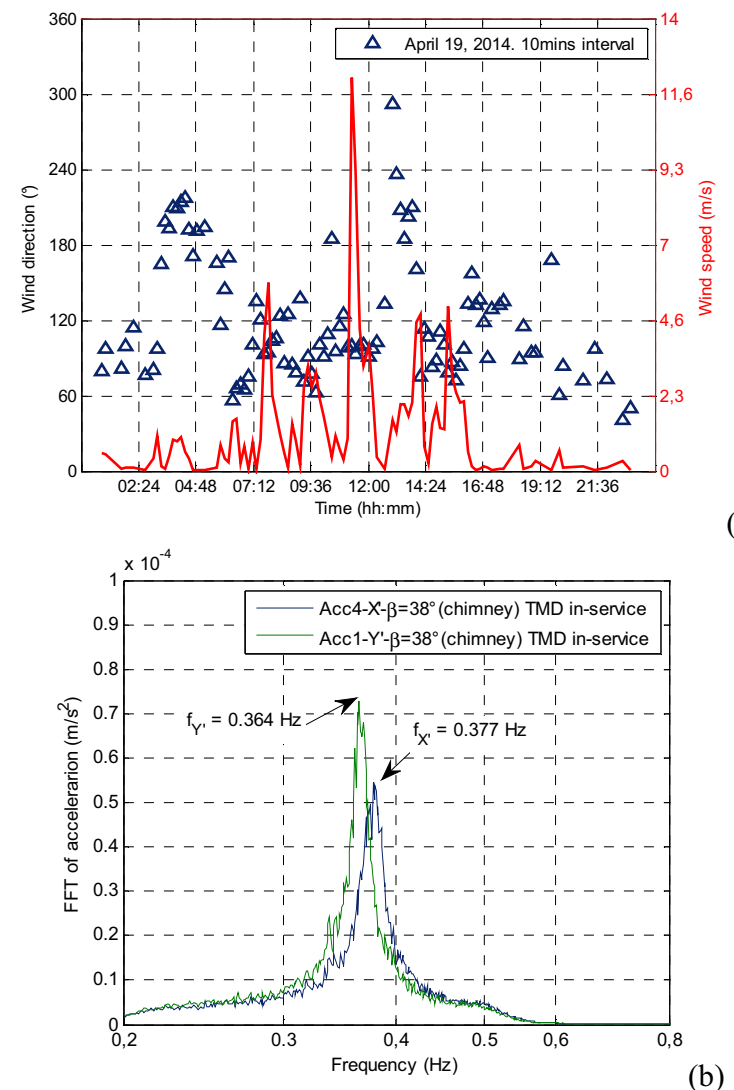

(a)

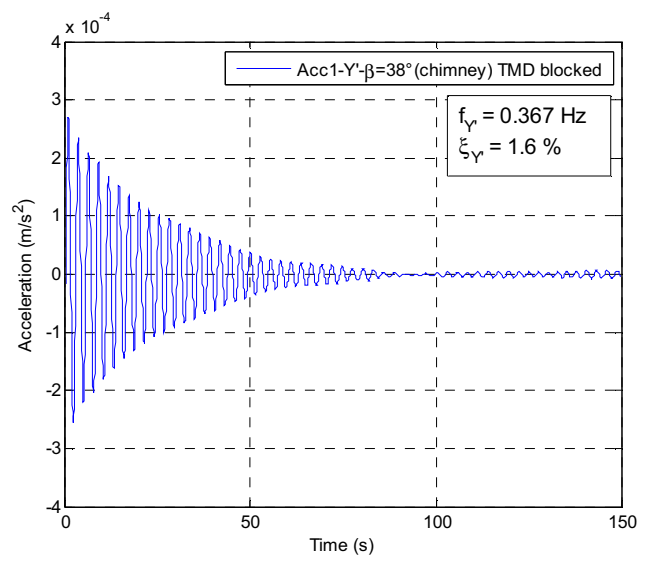

(c)

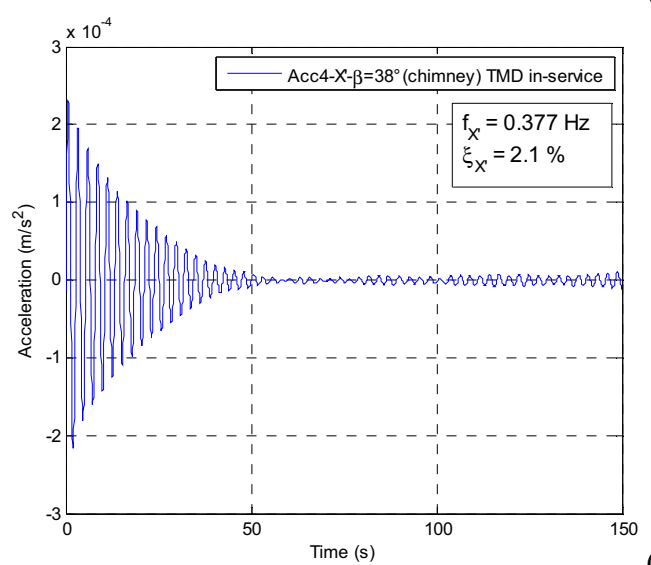

(d)

Figure 9. In-service results. (a) 30 mins interval mean wind speed and direction during the tests. (b) FFT of accelerometers on the chimney, reference system $X^{\prime}-Y^{\prime}, \beta=38^{\circ}$. (c-d) RD signatures of accelerometers Acc $1-Y^{\prime}$ and Acc $4-X^{\prime}$.
The analysis shows that whenever TMD is operating (in-service), this gives an increase in damping ratio to the first chimney mode from $\xi_{Y^{\prime}}=0.60 \%$ to $\xi_{Y^{\prime}}=1.60 \%$ in $Y^{\prime}$-direction and from $\xi_{X^{\prime}}=0.72 \%$ to $\xi_{X^{\prime}}=2.1 \%$ in $X^{\prime}$ direction giving an increasing of the chimney damping ratio by a minimum factor of 2.6 as an effect of the TMD, leading to an increase of the Scruton Number from $\mathrm{Sc}=16.8$ in the "TMD Blocked" configuration to $\mathrm{Sc}=37.8$ in the In-service configuration, well over the suggested CICIND value $\mathrm{Sc}=25$.

For the sake of completeness, it must be reported that this analysis was carried out with a single wind direction. Future elaborations will consider different wind conditions in terms of wind speed as well wind direction. In particular it will be very interesting to investigate possible interference effects between the two chimneys, i.e. instrumented chimney leeward and aligned to its twin counterpart.

\subsection{Contribution of the TMD}

Figure 10 shows the 10 minute time history of the accelerometers on the TMD $(A c c 2$ and $A c c 3)$; it is also shown the twin chimney direction which is basically coaligned with the wind direction. It can be noted that the predominant cycle shows a cross-direction acceleration, given by $A c c 3$, of about $0.067 \mathrm{~m} / \mathrm{s}^{2}$, which provides a maximum peak to peak displacement of the TMD of $25 \mathrm{~mm}$ at frequency $\mathrm{f}=0.366 \mathrm{~Hz}$. The Figure also shows the related spectra of the accelerometers Acc1 and Acc2, placed respectively on the tower and on the TMD.

The figure shows the peak associated to the first natural frequency of the coupled system, explicitly visible in both accelerometers. More specifically, it can be noted that the peak of the tower is about one-third smaller than the peak of related to the TMD, consistently with the effectiveness of the TMD.

\section{Future work}

The TMD installed on the chimney was designed to provide an easy and quick switch from the "TMD inservice" to the "TMD blocked" configuration. The full scale monitoring system can be upgraded with the purpose of providing a long term statistics. The aim of this work is also to provide a valuable contribution of knowledge from a quite unique full scale experiment about the twin cylinders interference effect, bringing possible cross-correlation between the structure response and wind direction and intensity, as well as the Scruton number of the chimney. The Rovinari full scale experiment could be then set up for long term monitoring in the various environmental conditions, representing a chance for full scale tests and validation of numerical models in this field.

\section{Conclusions}

Experimental tests were carried out on the chimney No. 3 at the Rovinari Power plant, Romania, in order to estimate the dynamic characteristic of the structure. Aim of the tests was also to verify the correct operation of the 

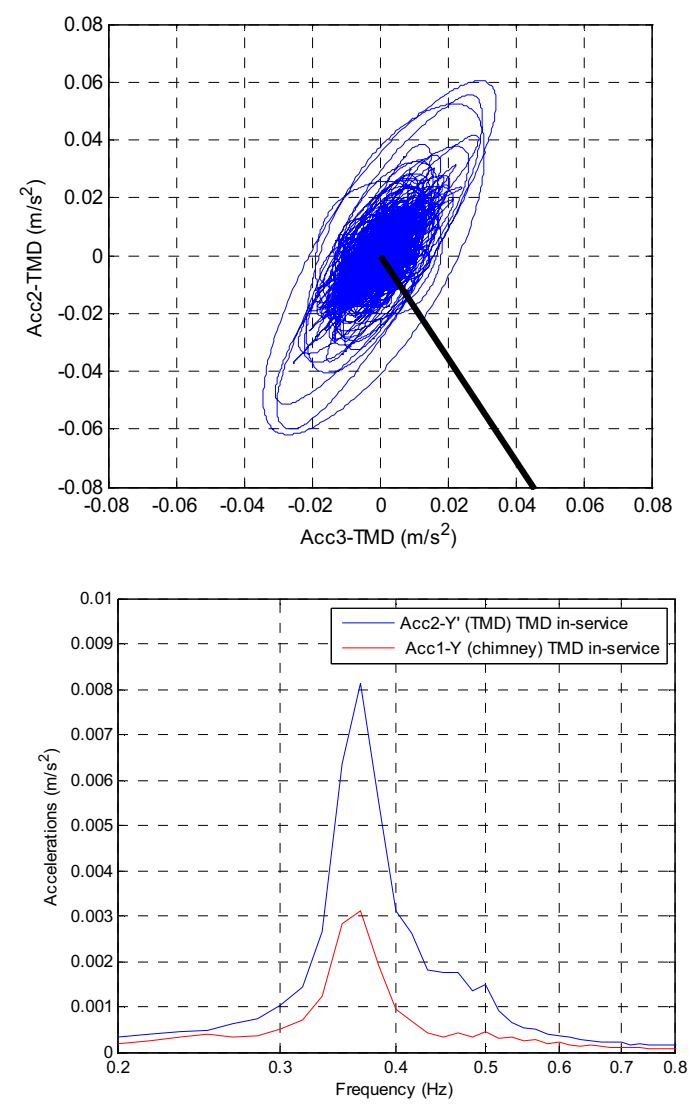

(b)

Figure 10. Acceleration values measured with wind speed $U_{\text {mean }}=11.8 \mathrm{~m} / \mathrm{s}$ and wind direction $\alpha \sim 110^{\circ}$. (a) Time history of accelerometers on the TMD (Acc2 and Acc3). The dotted line shows the direction of the twin chimney. (b) FFT of accelerometers in y-direction: Acc1 (chimney) and Acc2 (TMD).

TMD at the top of the chimney. For this purpose some measurements were done preventing the relative motion chimney-TMD through rigid constraints. The main results can be summarized as follows:

- The chimney showed a bisymmetrical structure because of two closely located dominant frequencies determined in the reference system $\mathrm{X}^{\prime}-\mathrm{Y}^{\prime}$ rotated with an angle $\beta=38^{\circ}$ respect the North-South direction. The estimated frequencies were equal to $f_{X^{\prime}}=0.403 \mathrm{~Hz}$ and $f_{Y^{\prime}}=0.382 \mathrm{~Hz}$, which differ about $5 \%$ from each other. It could be also noted that installation of the TMD resulted in a small reduction of the frequencies because of the considerable mass at the top of the chimney.

- The structural damping ratio of the first vibration mode of the chimney in the configuration "TMD blocked" (i.e. TMD rigidly connected to the chimney) was estimated equal to $\xi_{X^{\prime}}=0.72 \%$ and $\xi_{Y^{\prime}}=0.60 \%$. The structural damping was estimated along the principal axes of the chimney $\mathrm{X}^{\prime}-\mathrm{Y}^{\prime}$ in order to avoid beating phenomenon in the $\mathrm{RD}$ signature which invalidate the results.
- The first natural frequencies of the chimney in TMD in-service configuration were estimated equal $f_{X^{\prime}}=0.377 \mathrm{~Hz}$ and $f_{Y^{\prime}}=0.367 \mathrm{~Hz}$. It can be noted that the frequencies values were smaller than the previous configuration because of presence of the TMD inservice.

- The non-dimensioanl damping ratios along the principal axes were estimated equal to $\xi_{X^{\prime}}=2.1 \%$ and $\xi_{Y^{\prime}}=1.60 \%$, resulting in a Scruton Number respectively equal to $\mathrm{Sc}_{\mathrm{X}^{\prime}}=16.8$ and $\mathrm{Sc}_{\mathrm{Y}^{\prime}}=37.8$.

Long term and remote monitoring system, installed during this activity, will provide the authors also with further measurements used to investigate more thoroughly the interference effects and compared with the measurements in in-service configuration. Nevertheless, the data elaborated were useful to verify the correct operation of the TMD in increasing the damping of the coupled system and hence in reducing the vibration of the system, as well as systematically verifying the differences in the dynamics of design Vs built structures. Object of future works on the field could be carrying out simultaneously measurements on the adjacent chimney in a critical wind speed condition, trying also to study more accurately possible correlations among the response, wind, damping and frequencies.

\section{Acknowledgements}

The authors gratefully acknowledge the support given by Mr. Di Poi and the society CRI International as well as the International Committee on Industrial Chimneys (CICIND), for their very useful help and interest in this research activity .

\section{References}

1. N. Lin, C. Letchford, Y. Tamura, B. Liang and O. Nakamura, 'Characteristics of wind forces acting on tall buildings', Journal of Wind Engineering and Industrial Aerodynamics, 93 (2005): 217-242.

2. M. M. Zdravkovich, Flow around circular cylinders. 1: Fundamentals, (Oxford, 1997).

3. A. Simpson, 'On the flutter of a smooth circular cylinder in a wake', Aeronautical Quarterly, (1971): 25-41.

4. G. Diana, M. Belloli, S. Giappino, A. Manenti, L. Mazzola, S. Muggiasca and A. Zuin, 'Wind Tunnel Tests on Two Cylinders to Measure Subspan Oscillation Aerodynamic Forces', Power Delivery, IEEE Transactions on, 29 (2014): 1273-1283.

5. A. Zasso, M. Belloli, S. Giappino, and S. Muggiasca, "On the pressure and force field on a circular cylinder oscillating in the lock- in region at subcritical reynolds number", 9, pp. 919-927, 2006.

6. A. Zasso, M. Belloli, S. Giappino and S. Muggiasca, 'Pressure field analysis on oscillating circular cylinder', Journal of Fluids and Structures, 24 (2008): 628-650. 
7. M. Belloli, S. Giappino, S. Morganti, S. Muggiasca and A. Zasso, 'Vortex induced vibrations at high Reynolds numbers on circular cylinders', Ocean Engineering, 94 (2015): 140-154.

8. G. R. S. Assi, P. W. Bearman and J. R. Meneghini, 'On the wake-induced vibration of tandem circular cylinders: The vortex interaction excitation mechanism', Journal of Fluid Mechanics, 661 (2010): $365-401$.

9. S. O. Hansen and C. Dyrbye, Wind loads on structures, (Chichester, 1996).

10. CICIND - Tech. Rep, Model Code for Steel Chimneys, Revision 1 - 1999 Amendment A March 2002.

11. J. M. W. Brownjohn, E. P. Carden, C. R. Goddard and G. Oudin, 'Real-time performance monitoring of tuned mass damper system for a $183 \mathrm{~m}$ reinforced concrete chimney', Journal of Wind Engineering and Industrial Aerodynamics, 98 (2010): 169-179.

12. X. H. He, X. G. Hua, Z. Q. Chen and F. L. Huang, 'EMD-based random decrement technique for modal parameter identification of an existing railway bridge', Engineering Structures, 33 (2011): 13481356.

13. J. C. C. Asmussen, Modal Analysis Based on the Random Decrement Technique : application to civil engineering structures, Department of Mechanical Engineering, (1997).

14. P. Górski, 'Investigation of dynamic characteristics of tall industrial chimney based on GPS measurements using Random Decrement Method', Engineering Structures, 83 (2015): 30-49.

15. W.-X. Ren and Z.-H. Zong, 'Output-only modal parameter identification of civil engineering structures', Structural Engineering and Mechanics, 17 (2004): 1-16. 